\title{
Operative techniques in robotic thoracic surgery for inferior or posterior mediastinal pathology
}

\author{
Robert James Cerfolio, MD, FACS, FCCP, Ayesha S. Bryant, MSPH, MD, and \\ Douglas J. Minnich, MD, FACS
}

\begin{abstract}
Objective: Thoracic surgeons are performing robotic resections for anterior mediastinal tumors; however, tumors located in the posterior and especially the inferior chest can be difficult to approach robotically. The objective of this study was to evaluate the efficacy of the robot for resection of these tumors.
\end{abstract}

\begin{abstract}
Methods: We performed a retrospective review of the evolution and outcomes of our surgical technique for inferior or posterior mediastinal pathology.

Results: During a 30-month period, 153 patients underwent robotic surgery for pathology in the mediastinum, located in the inferior or posterior mediastinum in 75 of these patients. The most common indications for surgery were posterior mediastinal mass or lymph node in 41 patients, esophageal or bronchogenic cysts in 11 patients, esophageal leiomyoma in 7 patients, and diaphragmatic elevation in 7 patients. The median tumor size was 4.4 $\mathrm{cm}$, and the median length of stay was 1 day. One patient was converted to thoracotomy, but no patients were converted for bleeding. Morbidity occurred in 9 patients $(12 \%)$, major in 1 patient (a delayed esophageal leak after epiphrenic diverticulectomy). There was no mortality. Technical improvements included using robotic arm 3 posteriorly for retraction, side-docking, or coming over the back of the patient for tumors inferior to the inferior pulmonary vein and for diaphragmatic plication and using the lateral decubitus position for extraction of tumors larger than $3 \mathrm{~cm}$ via an access port over the tenth rib above the diaphragmatic fibers.
\end{abstract}

Conclusions: The robot affords safe access using a completely portal approach for resection of and surgical intervention for inferior and posterior chest pathology and for anterior tumors. Specific techniques can be used to improve the operation. (J Thorac Cardiovasc Surg 2012;143:1138-43)

A large number of cardiothoracic surgeons are quickly learning robotic surgery. ${ }^{1}$ The information reported by thoracic surgeons to the Society of Thoracic Surgeons' database reflects that a steadily increasing number of roboticassisted thoracic operations are being performed. ${ }^{2}$ Perhaps the greatest advantage that the robot offers the general thoracic surgeon is for the resection of mediastinal tumors and the intervention of other mediastinal pathology. Many studies have already demonstrated the safety of robotic thymectomy and the removal of other anterior mediastinal masses,

From the Division of Cardiothoracic Surgery, University of Alabama at Birmingham, Birmingham, Ala.

Disclosures: Dr Cerfolio: Ethicon, speaker/consultant; Neomend Inc, and Johnson \& Johnson Services Inc, consultant; Covidien, speaker; Precision, consultant, speaker, and grant recipient; Intuitive Surgical Inc, speaker and proctor. Dr Cerfolio receives money for each lecture he gives and for each day of robotic proctoring. In 2010 he proctored 7 surgeons, gave 18 lectures, and gave 32 lectures before surgery to 132 visiting observing surgeons at UAB. Dr Bryant has no financial disclosures to report. Dr Minnich is a consultant for Super Dimension.

Received for publication Sept 27, 2011; revisions received Nov 22, 2011; accepted for publication Dec 14, 2011; available ahead of print Jan 13, 2012.

Address for reprints: Robert James Cerfolio, MD, FACS, FCCP, Professor of Surgery, Chief of Thoracic Surgery, JH Estes Family Endowed Chair for Lung Cancer Research, Division of Cardiothoracic Surgery, University of Alabama at Birmingham, 703 19th St S, ZRB 739, Birmingham, AL 35294 (E-mail: rcerfolio@uab. edu).

$0022-5223 / \$ 36.00$

Copyright $(2012$ Published by Elsevier Inc. on behalf of The American Association for Thoracic Surgery

doi:10.1016/j.jtcvs.2011.12.021 most of which are located in the superior mediastinum. ${ }^{3-17}$ The patient positioning, port placement, and optimal location of the robot relative to the patient for anterior mediastinal masses and for thymectomy for myasthenia gravis are well described and reproducible. However, when the pathology is located in the inferior or posterior mediastinum, a robotic approach can be difficult. The optimal manner in which to position the patient and the robot remains poorly described for various location of tumors and pathology. ${ }^{18,19}$ We have implemented several technical changes in our operative technique to help overcome these challenges. The purpose of this study is to review and communicate these changes and how they have improved our results and decreased our operative time.

\section{MATERIALS AND METHODS}

This is a retrospective study using a prospective database of a consecutive series of patients who underwent a completely portal robotic operation for pathology in the inferior or posterior mediastinum using the da Vinci robotic system (Intuitive Surgical Inc, Sunnyvale, Calif). The details of our patient database and the methods used for obtaining follow-up data have been reported. ${ }^{20}$ Briefly, the data are entered into our database by 2 physicians (A.S.B. and R.J.C.) on the day of surgery. The database contains all of the variables collected by the Society of Surgeons' database and adheres to the same rigorous data validation process to ensure accuracy and quality assurance. In addition, we collect many other types of information, including genetic and molecular markers of tumors resected and 90-day mortality. 


\section{Abbreviations and Acronyms \\ CPRL-4 = completely portal robotic lobectomy using 4 arms \\ VATS $=$ video-assisted thoracoscopic surgery}

The entry criteria mandated that patients be at least 19 years old and have had a chest computed tomography scan within 40 days of surgery and an integrated positron emission tomography/computed tomography if indicated. There was no tumor characteristic (size or location) that was a contraindication to attempt to begin with a robotic approach in this series. Only pathology or tumors that were located in the inferior or posterior mediastinum were included in this study. Tumors in the anterior mediastinum and patients who underwent robotic Ivor Lewis esophagogastrectomy were excluded from this study. Tumor extraction was performed by placing the specimen in an anchor bag (Progressive Medical, Addison, Ill), de-docking the robot, and then removing it via the largest port used after enlarging that 1 portal incision.

At the time of surgery, the reasons to convert from a completely portal robotic operation to an open thoracotomy were bleeding that could not be controlled robotically, inability to enter the pleural space and insufflate carbon dioxide secondary to pleural symphysis from adhesions, or inability to completely remove the tumor and achieve an $\mathrm{R} 0$ resection.

The University of Alabama at Birmingham's Institutional Review Board approved this protocol (X100310009) and the prospective database used to collect information for this study (X030403013). Individual patient consent was waived for inclusion in this study; however, patient consent was obtained to enter patient information into the prospective database.

\section{Operative Technique}

If the mass or pathology is above the inferior pulmonary vein (whether it is anterior or posterior does not matter), our previously described operative technique ${ }^{21,22}$ is used for pulmonary resection, a completely portal robotic lobectomy using 4 arms (CPRL-4) (Figure 1). A classic lateral decubitus positioning is used for CPRL- 4 as opposed to a modified supine positioning, which is used for robotic thymectomy. This positioning and technique are selected when the tumor is greater than $3 \mathrm{~cm}$ and the tumor or pathology is located superior to the inferior pulmonary vein.

However, if the tumor is inferior to the inferior pulmonary vein, a different surgical approach is needed because the robot is unable to view this area well when it is driven in over the patient's head. A general principal is that the lesion to be resected or pathology that is to be operated on should lie in between the camera port and the robot. Thus, when a lesion is inferior in the chest the robot is side-docked alongside the operating room table (Figure 2). It should be driven in parallel to the patient, coming alongside of the patient's back, posteriorly. In this way, the surgeon can more easily view the diaphragm and the inferior and posterior chest. This mandates driving the robot in from the patient's feet. The robot's arms are not long enough to extend over the patient's feet as it can over the patient's head to reach trocars in the chest. Thus, it has to be side-docked. This technique is used for an inferior (in the lower one third of the esophagus) esophageal leiomyoma; any inferior posterior mediastinal tumor, such as a neurilemmoma on the ninth vertebral body or lower; esophageal myotomy for patients with achalasia; or diaphragmatic plication. It affords the surgeon a view of the entire mid to inferior aspect of the chest and allows the surgeon to work below the diaphragm if needed.

Another possible robotic positioning for tumors that are located in the inferior chest and the one we favor now is to place all the trocars in the mid-axillary line and drive the robot in dorsally over the patient's spine (Figure 3). We favor this for very low and posterior (not low and anterior)

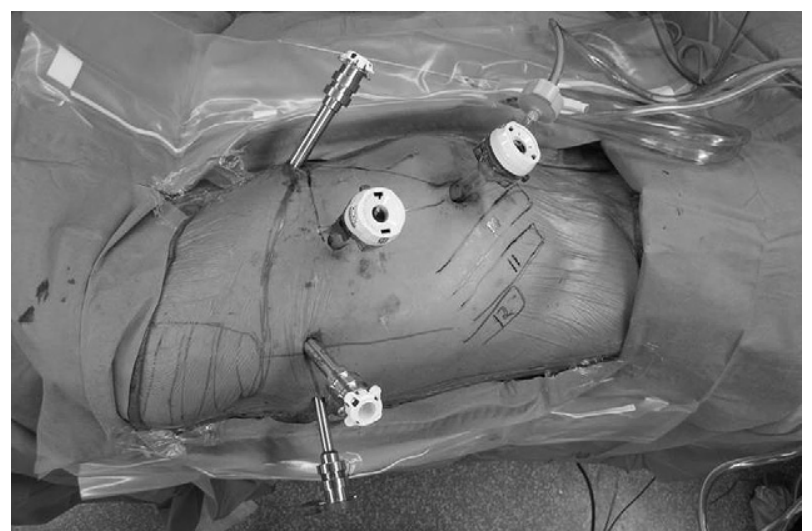

FIGURE 1. Intraoperative photograph, taken from above the patient in the left lateral decubitus position, depicts our operative technique currently used for right-sided posterior mediastinal tumors that are greater than 3 $\mathrm{cm}$ and located superior to the inferior pulmonary vein. The patient is placed in a classic left lateral decubitus position (with the right chest up and the head at the left side of the picture below). The trocar placements shown are also used to perform a CPRL-4.

diaphragmatic tumors because it is the operative technique we have used for robotic Ivor Lewis esophagogastrectomy operations. It has the advantage of being easier to drive the robot in over the patients' back than it is alongside the operating room bed.

Morbidity was defined using the definitions in the database (version 2.8) of The Society of Thoracic Surgeons. ${ }^{2}$ Operative mortality was defined as death within 30 days after surgery from any cause or before discharge. Tumor size reported was the largest diameter of the tumor as reported by the pathologist. Data were stored in Excel (Microsoft Corp, Seattle, Wash), and descriptive statistics are shown by the use of medians, standard deviations, and frequencies as appropriate.

\section{RESULTS}

Between March 1, 2009, and August 31, 2011, 153 patients underwent surgical intervention for pathology in the mediastinum; 78 of these patients underwent an operation for an anterior mediastinal mass, and 75 of these patients underwent intervention for an inferior or posterior mediastinal mass or pathology. Patients' characteristics are shown in Table 1. The overall median tumor size was $4.4 \mathrm{~cm}$ (range, 1-15.2 cm). The final diagnosis and intra- and postoperative outcomes are shown in Table 2. Complete (R0) resection was accomplished in all patients when a tumor was involved. The patient with the largest tumor $(15.2 \mathrm{~cm})$ had a lipoma that was soft and pliable, and it was easily removed via a relatively small port-like incision after being placed in a bag.

Postoperative morbidity occurred in 9 patients $(12 \%)$ (Table 2). One patient had a major complication: a delayed esophageal perforation. This occurred after a robotic epiphrenic diverticulectomy and myotomy. The patient was discharged the day after the operation after having a normal swallow that showed no leak. One week later, she returned with an empyema and the repeat swallow showed a small 


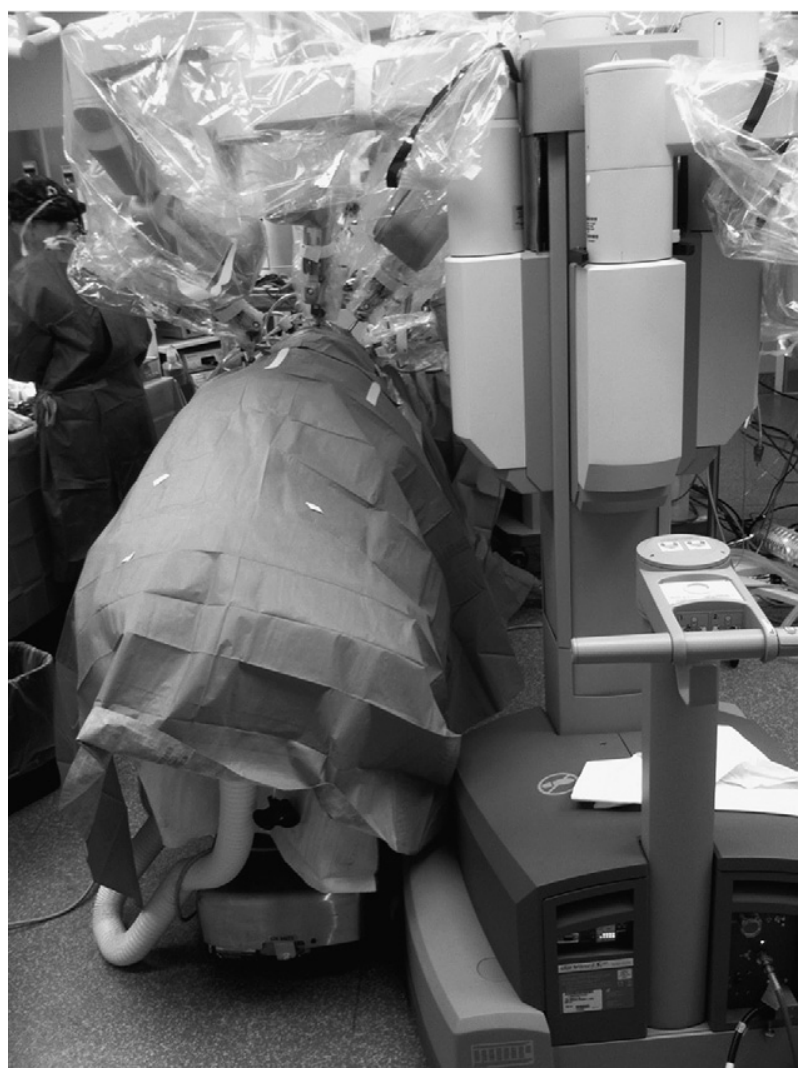

FIGURE 2. Intraoperative photograph depicts the robot coming alongside a patient's back, posteriorly, and being side-docked for a resection of a leftsided posterior and inferior located mediastinal mass.

4-mm esophageal leak. A stent was placed, and a left videoassisted empyectomy was performed. She made a full recovery but was in the hospital for 7 days.

There was 1 conversion from a robotic approach to a thoracotomy. This occurred in a patient who was undergoing resection of a large 7-cm-thick wall epiphrenic diverticulectomy and 2 smaller ones. The stapler tore the base of the large diverticulum, and we opened electively rather than try to re-staple it robotically.

There was no operative mortality. The median hospital length of stay was 1 day (range, $0-4$ days). Seven patients who had suspicious posterior lymph node or mass were discharged on the day of surgery. As shown in Table 3, the median operative time (defined as time from skin incision until skin closure) was 95 minutes (standard deviation \pm 21 minutes). The median operative times decreased progressively. The median operative time was 1 hour and 45 minutes for the first 25 patients, 1 hour and 32 minutes for the next 25 patients, and 1 hour and 16 minutes for the last 25 patients. The docking time (time from making the first incision to when the surgeon is sitting at the console and starts manipulating the robotic arms) also progressively decreased. The docking time was a median of $19 \pm 9$ minutes for the first 10 operations in this series, $14 \pm 5$

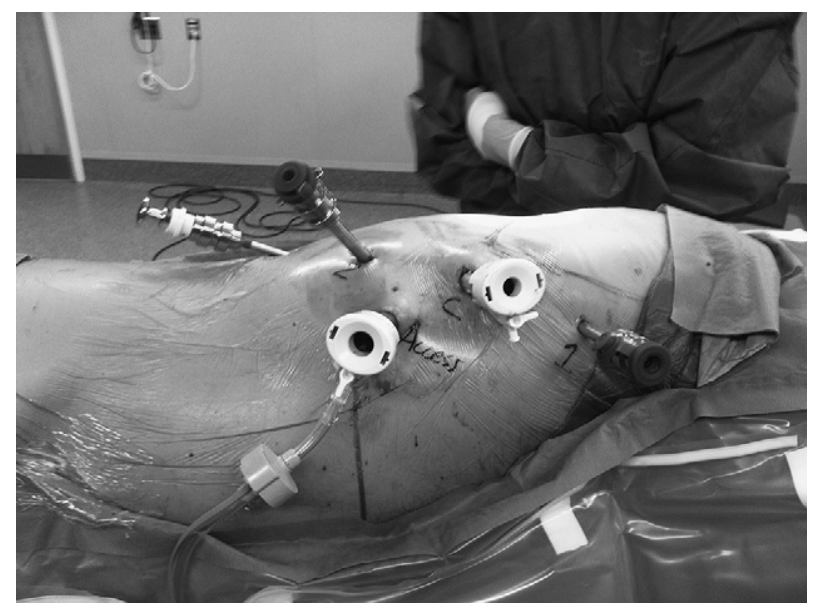

FIGURE 3. Intraoperative photograph depicts the trocar placement used for a right-sided robotic Ivor Lewis esophagogastrectomy. The ports are placed anteriorly, and the robot is not side-docked but rather driven in over the patient's back. This is also often used for diaphragmatic plication and low posterior tumors.

minutes for the next 10 procedures, and only $12 \pm 43$ minutes for the remaining 55 patients. Follow-up is complete in all patients with cancer, and none have developed a local or systemic recurrence.

\section{DISCUSSION}

The expanding use of robots in surgery is both controversial and undeniable. ${ }^{1}$ The capital cost of buying a robot, cost of maintenance, cost of equipment, need for credentialing, stapling of pulmonary vessels by bedside assistants during lobectomy, lack of palpation and limited haptic feedback, limited robotic availability for surgeons, and issues of proctoring all represent challenges to further adoption of robotic surgery. However, none change the fact that thoracic surgeons are now rapidly adding robotic surgery to their armamentarium, especially for operations in the mediastinum.

We recently presented our experience in starting a robotic program and stressed the need for obtaining the proper training required by the entire team. ${ }^{17}$ The results and safety of the use of the robot for resection of anterior mediastinal tumors, such as for thymoma, have been reported. ${ }^{23}$ However, surgeons have struggled with inferior or posterior mediastinal tumors. This is because when a CPRL-4 approach is chosen, the surgeon's view of the lower chest is limited. It is difficult and sometimes impossible to move the camera to see the diaphragm or operate on low posterior-inferior mediastinum tumors. Even taking down the inferior pulmonary ligament can be difficult with a CPRL-4 technique. This is true despite the fact that when we designed the CPRL-4, we moved the camera port to the seventh intercostal space, 2 spaces lower than it is located with a CPRL-3. In addition, significant adhesions between the lung and the diaphragm can also be troublesome with this port placement when 
TABLE 1. Characteristics of the 75 patients who underwent posterior mediastinal mass resection

\begin{tabular}{lc}
\hline & $\begin{array}{c}\text { Posterior mediastinal } \\
\text { mass resection } \\
\mathbf{N}=\mathbf{7 5}\end{array}$ \\
\hline Median age in years, $\pm \mathrm{SD}$, (range) & $50 \pm 18(20-88)$ \\
Gender & \\
Male & $37(49 \%)$ \\
Female & $38(51 \%)$ \\
Race & \\
White & $68(91 \%)$ \\
Black & $7(9 \%)$ \\
Height (inches), median & $68 \pm 9$ \\
Weight (lbs), median & $187 \pm 14$ \\
Body mass index, median & $28.4 \pm 3.2$ \\
Comorbidities & \\
Hypertension & $32(43 \%)$ \\
Chronic steroid use & $4(5 \%)$ \\
Heart disease or heart failure & $18(24 \%)$ \\
Prior cardiothoracic surgery & $4(5 \%)$ \\
Cerebral vascular accident/stroke & $3(4 \%)$ \\
Diabetes & $15(20 \%)$ \\
COPD & $5(7 \%)$ \\
ECOG (median) & $1(0-3)$ \\
\hline ECOG, Eastern Cancer Oncology Group; $S D$, standard deviation; $C O P D$, chronic \\
obstructive pulmonary disease.
\end{tabular}

the robot is driven over the patient's head. Thus, a CPRL-4 is not the best technique to select for inferior or posterior tumors. There remains significant confusion as to the ideal patient positioning and the best direction to drive the robot for these patients. The current study has shown the safety and efficacy of robotic operations for these tumors and relates our experience using different operative techniques.

\section{Technical Lessons Learned}

Inferior or posterior mediastinal tumors can be difficult to remove robotically or using video-assisted thoracoscopic surgery (VATS). Most VATS operations are performed from anterior to posterior. The ribs are closer together posteriorly, and thus some surgeons have found it difficult to place their fingers into the chest or to insert ports posteriorly. The reason that so many adroit VATS surgeons are now using robotic techniques for their mediastinal operation is the advantages the robot provides: It has wristed instruments and improved optics, the surgeon drives the camera, it affords the ability to navigate and see more parts of chest, and it is ideal for confined narrow spaces, such as the mediastinum or pelvis. However, viewing the inferior and posterior mediastinum when the robot is driven in over the patient's head (as used most commonly for robotic pulmonary resections) is also problematic. However, if the robot is driven in from a posterior location and the camera is placed anteriorly, it becomes easier. Because this operation and set-up are used infrequently, many robotic teams
TABLE 2. Final pathology/diagnosis and intraoperative and postoperative outcomes

\begin{tabular}{|c|c|}
\hline Location & $\begin{array}{c}\text { Posterior } \\
\text { mediastinal resection } \\
\mathrm{N}=75 \\
\end{array}$ \\
\hline \multicolumn{2}{|l|}{ Final pathology/diagnosis } \\
\hline \multicolumn{2}{|l|}{ Posterior mediastinal masses } \\
\hline Ganglioneuroma & 2 \\
\hline Neurofibroma & 1 \\
\hline Plasmacytoma & 1 \\
\hline Schwannoma & 3 \\
\hline Thymoma, posterior, recurrent & 3 \\
\hline Large lipoma of mediastinum & 1 \\
\hline \multicolumn{2}{|l|}{ Cyst } \\
\hline Bronchogenic cyst & 8 \\
\hline Esophageal duplication cyst & 3 \\
\hline \multicolumn{2}{|l|}{ Lymph nodes, malignant } \\
\hline Metastatic cancer & 23 \\
\hline Lymphoma & 2 \\
\hline \multicolumn{2}{|l|}{ Lymph nodes, benign } \\
\hline Caseating granuloma & 4 \\
\hline Mycobacterium tuberculosis & 1 \\
\hline \multicolumn{2}{|l|}{ Others } \\
\hline Esophageal leiomyoma & 7 \\
\hline Diaphragmatic elevation & 7 \\
\hline Epiphrenic diverticulum & 5 \\
\hline Achalasia failed abdomen approach & 3 \\
\hline Bochdalek diaphragmatic hernia & 1 \\
\hline Estimated blood loss, median (range) & $50 \mathrm{~mL}(25-175)$ \\
\hline $\begin{array}{l}\text { Conversion from robotic to open } \\
\text { operation approach }\end{array}$ & 1 \\
\hline Hospital length of stay (d), median & $1(0-4)$ \\
\hline \multicolumn{2}{|l|}{ Morbidity } \\
\hline Atrial fibrillation & 4 \\
\hline Pneumothorax & 2 \\
\hline Gout & 1 \\
\hline Prolonged air leak & 1 \\
\hline Esophageal leak (delayed)* & 1 \\
\hline
\end{tabular}

are unaware of it. We struggled with many of these issues initially and suffered instrument collisions and an inability to fully view and work on pathology in this location until we started to drive the robot in over the patient's back and place the ports anteriorly.

This report shows that the robot affords the safe and complete removal of inferior, posterior, and even large mediastinal tumors. If the tumor is large $(>3 \mathrm{~cm})$, the patient should be positioned in a lateral decubitus position and even tilted forward. This allows the lung and blood to fall away from the operative target, better exposing the posterior mediastinum. Although some have used prone positioning for esophageal procedures or operations on the posterior mediastinum, we no longer favor it because we prefer a double-lumen endotracheal tube. In our experience, placing a patient on his/her abdomen with 
a double-lumen endotracheal tube often adds too much to the anesthetic set-up time because most anesthesiologists favor a head-plate to protect the patient's palate from the double-lumen tube. We therefore prefer the lateral decubitus position and then lean the patient anteriorly, which is much quicker. The operating room team is usually more comfortable with this positioning, and this is faster and easier to do and still affords the blood and lung to fall away from the operative field.

We prefer the use of the fourth robotic arm for these operations, just as we have described its usefulness for the CPRL-4 operation. ${ }^{18}$ It allows the surgeon to retract the arm and reduces the moves and skill required by the bedside assistant. The $15-\mathrm{mm}$ non-robotic port serves as the entry point for rolled-up sponges or a sucker if needed, which are placed by the bedside assistant. It is also where the tumor is removed after it is placed in a bag. However, if the procedure is for a resection of an esophageal leiomyoma, this fifth incision is often not needed.

If the targeted lesion is at the level of the inferior pulmonary vein or inferiorly and posterior, it is best to place all the trocars anteriorly in the mid or anterior axillary line and then bring the robot in from the patient's back as used for robotic esophagectomy (Figure 3). This positioning allows an unimpeded view of the entire surgical field.

If the desired operation is to plicate the diaphragm, remove an anterior tumor on the diaphragm, or repair an anterior diaphragmatic hernia, then the optimal set-up is anterior port placement and the robot is driven in over the patient's back (Figure 3). Another option, but now our second choice, is a "superior CPRL-4" port placement. In this technique the trocars are placed higher than the standard CPRL-4. The ports are placed over the fifth or sixth rib, and not over the seventh or eighth rib as we do for a CPRL-4 for pulmonary lobectomy. The access port is placed superiorly over the third or fourth rib, and not inferiorly over the tenth rib as it is done for a lobectomy. This is because the robot is side-docked, meaning it is driven in from below and positioned parallel to the operating room alongside the patient's back (Figure 2). By sidedocking, the target now becomes the inferior chest and diaphragm. This operation can be technically difficult, and we have had some difficulties with the robotic arm 2 (the left hand) reaching the anterior aspect of the diaphragm. Most recently, we have used the approach shown in Figure 3 and also prefer it for diaphragmatic plication. Further data and experience are needed to recommend a robotic approach for patients with a thick-walled or large epiphrenic diverticulum.

Study limitations are that it is uni-institutional, it features only 1 surgeon, and there is no $P$ value to statistically validate our findings. Rather, it is a collection of lessons learned by us from attempting to perform these operations in many different ways. The strengths of the study are the large number of patients, the diverse pathology, and the various anatomic locations, thus making the findings of this study helpful to many different types of operations and patients.

\section{CONCLUSIONS}

We have shown that many different types of inferior or posterior mediastinal pathology can be safely performed, approached, handled, resected, or sewn using robotic techniques. There are a plethora of technical maneuvers that can help speed robotic surgery, reduce the frustration of learning robotics, and improve its safety for patients. If robotic surgery is to continue to grow both in and outside of the United States, techniques that further reduce costs, operative times, and morbidity are needed. In addition, further studies that evaluate outcomes and demonstrate the advantages or disadvantages of VATS and robotic techniques are needed, especially in patients with cancer.

\section{References}

1. Intuitive Surgical. Intuitive Surgical Web site. Available at: http://www. intuitivesurgical.com. Accessed November 18, 2011.

2. Society of Thoracic Surgeons. STS national database. Available at: http://www. sts.org/national-database. Accessed October 1, 2011.

3. Augustin F, Schmid T, Sieb M, Lucciarini P, Bodner J. Video-assisted thoracoscopic surgery versus robotic-assisted thoracoscopic surgery thymectomy. Ann Thorac Surg. 2008;85:S768-71.

4. Savitt MA, Gao G, Furnary AP, Swanson J, Gately HL, Handy JR. Application of robotic-assisted techniques to the surgical evaluation and treatment of the anterior mediastinum. Ann Thorac Surg. 2005;79:450-5

5. Kernstine KH. Robotics in thoracic surgery. Am J Surg. 2004;188:89-97.

6. Rea F, Marulli G, Bortolotti L, Feltracco P, Zuin A, Sartori F. Experience with the "da Vinci" robotic system for thymectomy in patients with myasthenia gravis: report of 33 cases. Ann Thorac Surg. 2006;81:455-9.

7. Yoshino I, Hashizume M, Shimada M, Tomikawa M, Tomiyasu M, Suemitsu R, et al. Thoracoscopic thymectomy with the da Vinci computer enhanced surgical system. J Thorac Cardiovasc Surg. 2001;122:783-5.

8. Rueckert JC, Ismail M, Swierzy M, Sobel H, Rogalla P, Meisel A, et al. Thoracoscopic thymectomy with the da Vinci robotic system for myasthenia gravis. Ann NY Acad Sci. 2008;1132:329-35.

9. Bodner J, Wykypiel H, Greiner A, Kirchmayr W, Freund MC, Margreiter R, et al. Early experience with robot-assisted surgery for mediastinal masses. Ann Thorac Surg. 2004;78:259-65.

10. Yoshino I, Hashizume M, Shimada M, Tomikawa M, Sugimachi K. Video-assisted thoracoscopic extirpation of a posterior mediastinal mass using the da Vinci computer enhanced surgical system. Ann Thorac Surg. 2002;74:1235-7.

11. Morgan JA, Ginsburg ME, Sonett JR, Morales DL, Kohmoto T, Gorenstein LA, et al. Advanced thoracoscopic procedures are facilitated by computer-aided robotic technology. Eur J Cardiothorac Surg. 2003;23:883-7.

12. Morgan JA, Kohmoto T, Smith CR, Oz MC, Argenziano M. Endoscopic computer-enhanced mediastinal mass resection using robotic technology. Heart Surg Forum. 2003;6:E164-6.

13. Bodner JC, Zitt M, Ott H, Wetscher GJ, Wykypiel H, Lucciarini P, et al. Roboticassisted thoracoscopic surgery (RATS) for benign and malignant esophageal tumors. Ann Thorac Surg. 2005;80:1202-6.

14. DeRose JJ, Swistel DG, Safavi A, Connery CP, Ashton RJ Jr. Mediastinal mass evaluation using advanced robotic techniques. Ann Thorac Surg. 2003;75: 571-3.

15. Elli E, Espat NJ, Berger R, Jacobsen G, Knoblock L, Horgan S. Robotic-assisted thoracoscopic resection of esophageal leiomyoma. Surg Endosc. 2004;18: 713-6.

16. Augustin F, Schmid T, Bodner J. The robotic approach for mediastinal lesions. Int J Med Robot. 2006;2:262-70.

17. Castle SL, Kernstine KH. Robotic-assisted thymectomy. Semin Thorac Cardiovasc Surg. 2008;20:326-31. Review. 
18. Al-Mufarrej F, Margolis M, Tempesta B, Strother E, Gharagozloo F. Novel thoracoscopic approach to difficult posterior mediastinal tumors. Gen Thorac Cardiovasc Surg. 2010;58:636-9.

19. Morgan JA, Ginsburg ME, Sonett JR, Morales DL, Kohmoto T, Gorenstein LA, et al. Advanced thoracoscopic procedures are facilitated by computer-aided robotic technology. Eur J Cardiothorac Surg. 2003;23:883-7.

20. Bryant AS, Rudemiller K, Cerfolio RJ. The 30- versus 90-day operative mortality after pulmonary resection. Ann Thorac Surg. 2010;89:1717-23.
21. Cerfolio RJ, Bryant AS, Minnich DJ. Starting a robotic program in genera thoracic surgery-why, how and lessons learned. Ann Thorac Surg. 2011;91: 1729-37.

22. Cerfolio RJ, Bryant AS, Skylizard L, Minnich DJ. Initial consecutive experience of completely portal robotic pulmonary resection with 4 arms. $J$ Thorac Cardiovasc Surg. 2011;142:740-6.

23. Limmer KK, Kernstine KH. Minimally invasive and robotic-assisted thymus resection. Thorac Surg Clin. 2011;21:69-83. 